\title{
Planetary nebula abundances in dwarf galaxies
}

\author{
Denise R. Gonçalves \\ Valongo Observatory - Federal University of Rio de Janeiro \\ Ladeira Pedro Antonio 43, Rio de Janeiro, Brazil \\ email: denise@astro.ufrj.br
}

\begin{abstract}
The framework of the present review is as follows. The Local Group (LG) is populated by dwarf galaxies belonging to different morphological types. Apparently these galaxies are very different. The questions naturally raised from this framework are many. Is there an evolutionary sequence among them? Do they share common progenitors? Is the environment at the origin of their differences? The method we propose to answer to these questions is by investigating the chemical evolution of dwarf galaxies and their mass-metallicity relation (MZR). To this aim we use metallicities derived from planetary nebulae, since this stellar population is present in the star-forming (dwarf irregular, dIrr) as well as in the quiescent (dwarf spheroidal, dSph) galaxies. The results, actually, show that both dIrr and dSph galaxies of the LG follow the same MZR, at variance with the differences claimed in the past. These results are in good agreement with the recently derived MZR, based on stellar instead of the nebular metallicities of the LG dwarf galaxies. Moreover, our MZR is also consistent with the global MZR of SLOAN star-forming galaxies, which spans a wider stellar mass range $\left(\sim 10^{6}-10^{11} \mathrm{M}_{\odot}\right)$ than the LG dwarfs.
\end{abstract}

Keywords. ISM: abundances, planetary nebulae; galaxies: dwarf, abundances, evolution.

\section{Local Group dwarf galaxies}

- The LG dwarf irregular galaxies - dIrrs: are HI-dominated; with variety of irregular shapes. They are found in galaxy clusters, small galaxy groups, and in the field, tending to be isolated. They are also characterised by having almost constant star formation rate (Zhang et al. 2012), and old stellar populations have been detected in almost all dIrrs.

- The LG dwarf spheroidal galaxies - dSphs: are dynamically evolved stellar systems; with intermediate to old age stellar populations. These galaxies have mild rotation, and are dominated by dark matter. In the LG, they are typically found close to Andromeda (M31) or to the Milky Way (MW). Though almost devoid of gas in their central parts, some evidence for gas has been found, e.g., in NGC 185 (Gonçalves et al. 2012).

- The LG dwarf elliptical galaxies - dEs: they have regular shape and are not completely depleted of gas, being also rotationally supported. In the LG all dEs are located around M31, NGC 205 being the best example (Monaco et al. 2009). They pose a mixture of stellar populations and complex star formation histories (Carraro et al. 2001).

In terms of total (and HI) mass, the dEs, dSphs and dIrrs have, respectively, $\leqslant 10^{8}$, $\leqslant 10^{5}$ and $\leqslant 10^{9} \mathrm{M}_{\odot}\left(\leqslant 10^{9}, \sim 10^{7}\right.$ and $\left.\leqslant 10^{10} \mathrm{M}_{\odot}\right)$ (Carraro 2014).

\section{The [morphological] evolution of the [dwarf] galaxies}

Kormendy \& Djorgovski (1989) proposed an evolutionary relation between the two main morphological types of dwarfs, on which only one type of dwarf galaxy existed when the LG formed, and then the dSphs were formed through the removal of gas in 


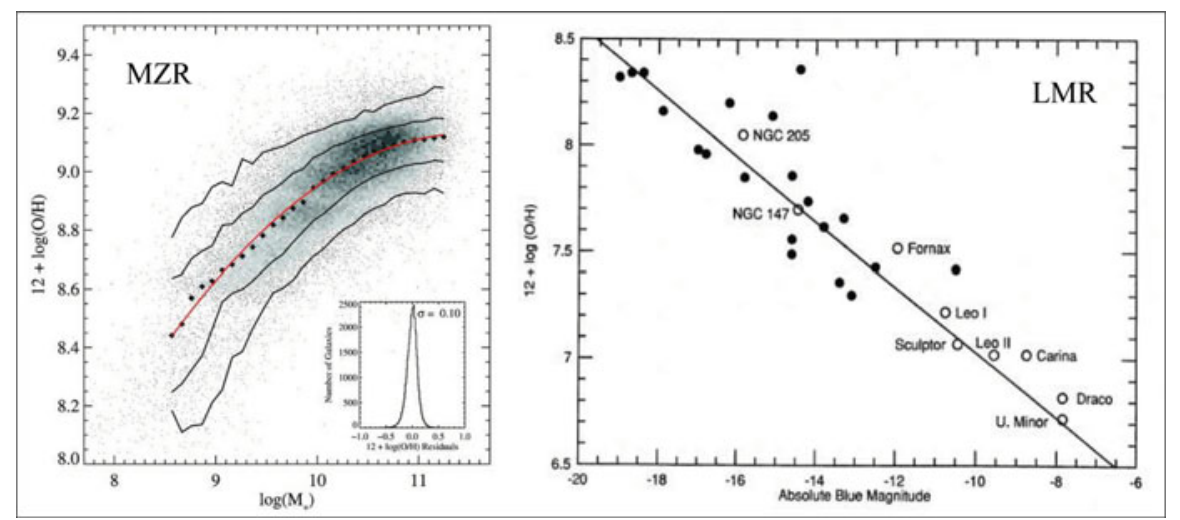

Figure 1. Left: Tremonti et al. (2004) mass-metallicity relation, on which a tight correlation between $\mathrm{M}_{*}$ and $\mathrm{Z}$ is found from the SDSS spectroscopy of 53,000 star-forming galaxies at $\mathrm{z}$ 0.1. Right: Skillman et al. (1989) luminosity-metallicity relation for the dIrrs (filled symbols) and dSphs (empty symbols). For the latter galaxies, the $\mathrm{O} / \mathrm{H}$ is a conversion from the $[\mathrm{Fe} / \mathrm{H}]$ derived from the old stellar population.

dIrrs (either via ram-pressure stripping, supernova-driven winds or star formation). More recently Kormendy \& Bender (2012) review the morpho-evolution of galaxies in general, not changing significantly this view of dwarfs evolution. Indeed, all dwarfs show signature of old stellar populations, although in different amounts (Weisz et al. 2014). These ideas imply that all dwarfs started to form stars at a sharply defined early epoch.

What is the insight the mass-metallicity relation (MZR) could give to the morphological dwarf galaxy evolution? Stellar mass reflects the amount of gas locked up into stars. Metallicity $(\mathrm{Z})$, on the other hand, reflects the gas reprocessed by stars and any exchange of gas between the galaxy and its environment. Tremonti et al. (2004) found a tight correlation between stellar mass $\left(\mathrm{M}_{*}\right)$ and $\mathrm{Z}$ spanning over 3 orders of magnitude in $\mathrm{M}_{*}$ and a factor of 10 in $\mathrm{Z}$, as it can be seen in Fig. 1, adapted from Fig. 6 in Tremonti et al. (2004). For this plot, authors considered 53,000 SDSS star-forming galaxies. It is important to also note that the metallicities used in the plot are the oxygen abundances $(\mathrm{O} / \mathrm{H})$ derived from the $\mathrm{H}$ II regions of the galaxies. The interplay of different effects -star formation efficiency, stellar winds, outflows/inflows and the depth of the potential wells- is responsible for this tight correlation.

A long time ago, in fact back in 1989, Skillman et al. (1989) found a clear relation between the $\mathrm{O} / \mathrm{H}$ and the absolute magnitude of a number of nearby dwarf irregulars. Actually, this paper also shows that the dwarf ellipticals adhere to the same abundanceluminosity relation (LZR) followed by the dIrrs (see the right panel of Fig. 1, which was extracted from Fig. 2 of Skillman et al. (1989). These authors pointed out the fact that the relation just discussed could be taken as a support of the 'theory' that dwarf ellipticals are formed by stripping of gas of the dIrrs.

In this scenario, as described by Richer \& McCall (1995), once the gas finished and the star formation stopped, the old dwarf irregular-like galaxies fade in luminosity as their stellar population ages. Therefore, dSphs are expected to have, at a given luminosity, higher metallicities than dIrrs.

\section{The need of nebular abundances from $\mathrm{PNe}$}

The metallicity of the star-forming dIrrs is obtained by measuring the $\mathrm{O} / \mathrm{H}$ of their $\mathrm{H}$ II regions. In dSphs - dominated by the old stellar population - the derived value are 

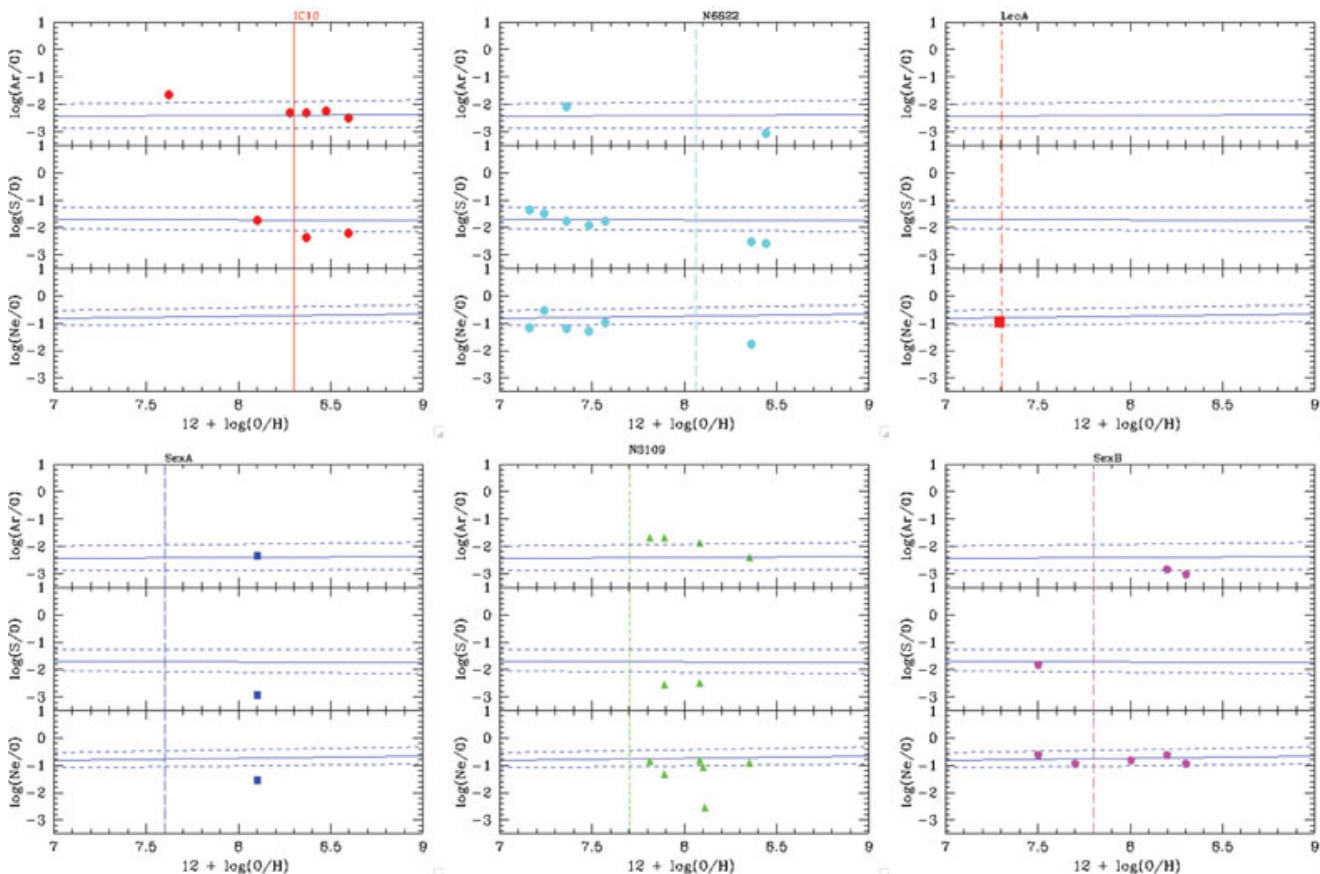

Figure 2. $\mathrm{PNe} \mathrm{Ne} / \mathrm{O}, \mathrm{S} / \mathrm{O}$ and $\mathrm{Ar} / \mathrm{O}$ abundance ratios versus oxygen abundance in $\mathrm{IC} 10$, NGC 6822, Leo A, Sex A, NGC 3109 and Sex B, nearby dIrrs. Symbols are: red circles for IC10; blue circles for NGC 6822; red box for Leo A; blue box for Sex A; green triangles for NGC 3109; and magenta losangles for Sex B. The quasi-horizontal lines show the abundance line ratios given by the sample H II regions of blue compact galaxies, as compiled by Izotov et al. (2006). The vertical lines show the $12+\log (\mathrm{O} / \mathrm{H})$ of the $\mathrm{H}$ II regions of each of the dIrr in this plot. These lines are labelled with the corresponding galaxy identification at the top of the plot.

the $[\mathrm{Fe} / \mathrm{H}]$ from stars. Assumptions about the $[\mathrm{O} / \mathrm{Fe}]$ are very uncertain, since this ratio depends on the star formation history. Thus, Richer \& McCall (1995) proposed the use of metallicities derived from PNe that are present in the star-forming as well as in the quiescent dSph galaxies.

In contrast to $\mathrm{H}$ II regions, some elemental abundances in $\mathrm{PNe}$ are affected by the nucleosynthesis in the progenitor stars, since newly synthesized material can be dredged-up by convection in the envelope, significantly altering the abundances of $\mathrm{He}, \mathrm{C}$ and $\mathrm{N}$ in the surface layers during the giant branch and asymptotic giant branch (AGB) phases. And, following Kingsburgh \& Barlow (1994), Péquignot et al. (2000) and Leisy \& Dennefeld (2006) a certain amount of oxygen can be mixed in during the thermally pulsing phase of the AGB evolution. However, the dredge-up of $\mathrm{O}$ and Ne seems a rare event, happening mainly in low-metallicity environments, therefore we believe that oxygen is a good tracer of the ISM abundances at the epoch of the PN progenitor formation. Various studies have analysed the circumstances that make the dredge-up of oxygen possible, both from theoretical and observational points of view (Siess et al. 2004; Magrini et al. 2005a; Richer \& McCall 2007; Kniazev et al. 2005; Peña et al. 2007; Kniazev et al. 2007, 2008).

In Fig. 2, we study the presence or not of the third-dredge in a number of nearby dwarf irregular galaxies. We do not plot the data for dwarf spheroidal galaxies since the comparison with the present-time ISM is not possible. The idea behind the comparison of $\mathrm{PNe}$ abundance ratios as a function of the oxygen abundance in different galaxies, 
with the $12+\log (\mathrm{O} / \mathrm{H})$ of the $\mathrm{H}$ II regions in each of these galaxies (vertical lines), and in a sample of blue compact galaxies (BCGs, 'horizontal' lines), is that in H II regions, the oxygen that is seen has been produced by the same massive stars that produced the $\alpha$-process neon, sulphur and argon. Therefore, in these plots, $\log (\mathrm{Ne} / \mathrm{O}), \log (\mathrm{S} / \mathrm{O})$ and $\log (\mathrm{Ar} / \mathrm{O})$ should be constant and show no dependence on the oxygen abundance. In fact, inspecting Fig. 2, we see that in the three abundance ratios most of the PNe are distributed along the line that defines the BCGs abundance ratios of $\mathrm{H}$ II regions, thus with no trends with respect to the oxygen abundance. The oxygen production in these diagrams should have two effects: (i) to place the $\mathrm{PN}$ points well ahead of the mean abundance in the ISM, (ii) to depress the Ne/O, S/O and $\mathrm{Ar} / \mathrm{O}$ ratios, unless the production of $\mathrm{Ne}, \mathrm{S}$ and $\mathrm{Ar}$ also occurs. In a conservative view, we would expect that the presence of both effects would probe the third-dredge-up of $\mathrm{O}$. Few PNe pass this test full-filling both requests, usually with abundance ratios that are not completely understood. Note, from the plots for galaxies of much lower metallicity, that this contribution is important in the case of Sex A $[12+\log (\mathrm{O} / \mathrm{H})=7.6]$ and NGC $3109[12+\log (\mathrm{O} / \mathrm{H})$ $=7.7$. Moreover, the plots in Fig. 2 also give us a lower limit above of which no third dredge-up effect is seen in the nearby dwarf irregulars. We conclude that this limit is around $12+\log (\mathrm{O} / \mathrm{H})=7.7$ (Magrini \& Gonçalves 2009).

\section{Dwarf galaxies' luminosity- and mass-metallicity relations}

Given the controversial existence of an offset between the LZR of dwarf galaxies, in the sense that for a given luminosity higher metallicities would be found for the dSphs as compared with the dIrrs, we re-analysed the PN LZR and the MZR of the LG dwarfs, in Fig. 3.

To build the LZR the $\mathrm{O} / \mathrm{H}$ come from our studies and the literature (see the figure caption), whereas the luminosities $\left(\mathrm{M}_{B}\right)$ are from Mateo (1998), Lee et al. (2003) and van den Bergh (2007). The continuous line shown in Fig. 3 (left panel) gives the weighted least-squares fit to the $\mathrm{Z}$ versus luminosity data for the 50 dIrrs within a distance of $5 \mathrm{Mpc}$, as in van Zee et al. (2006). First note that the location of the dIrrs and dSphs in the LZR cannot be clearly separated from each other, meaning that the offset we found previously (see Richer \& McCall 1995 and Gonçalves et al. 2007) has vanished. This is so because in the present version of the plot we use new (and more accurate) measurements of PN metallicities. Coherently, this is in agreement with the latest results from the spectroscopically determined stellar LZR (Kirby et al. 2013).

As discussed in the previous section, $\mathrm{O}$ was produced, and brought to the surface of the central stars via the third dredge-up in a few PNe of the dIrrs Sex A (Magrini et al. 2005) and NGC 3109 (Peña et al. 2007). Judging from Fig. 3, the presence or not of these two galaxies would not change the LZR significantly. Following Kniazev et al. (2007), the self-production of $\mathrm{O}$ occurred in the Fornax. A lower $12+\log (\mathrm{O} / \mathrm{H})$, by $0.27 \pm 0.10$, was proposed by these authors in order to reconcile the galaxy abundance patterns of $\mathrm{S} / \mathrm{O}$, $\mathrm{Ne} / \mathrm{O}$ and $\mathrm{Ar} / \mathrm{O}$ with the expected values. The other discrepant dwarf in the relation is Sgr, whose contamination via accretion of enriched gas expelled by our Galaxy could better explain its PNe O/H (Zijlstra et al. (2006).

The mass-metallicity relation (MZR) of the LG dwarf galaxies is given in the right panel of Fig. 3. We note that, as in the case of the LZR, dIrrs and dSphs are not segregated, and follow a common relation. In this figure we plot the least-squares fit obtained by Kirby et al. (2013, see their eq. 4) using the stellar metallicities in dwarf galaxies and our least mean square fit (see Gonçalves \& Magrini 2014 for the details of the conversion). The lowest mass in Kirby's et al. (2013) was of the order of $10^{3} \mathrm{M}_{\odot}$. In Fig. 3, we encompass a 

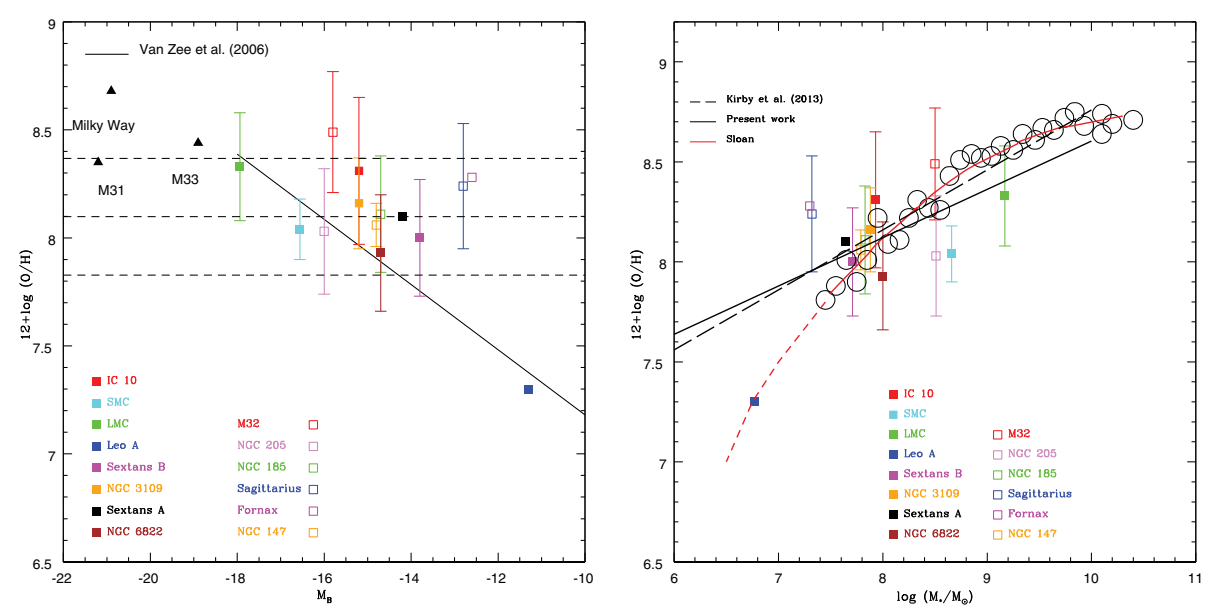

Figure 3. Left: The PNe LZR of the LG dwarf galaxies, showing $12+\log (\mathrm{O} / \mathrm{H})$ vs. magnitude. Filled symbols represent the dIrrs while the dSphs are have empty symbols. The continuous line represents the LZR, from $\mathrm{H}$ II regions of nearby $(\mathrm{D} \leqslant 5 \mathrm{Mpc})$ galaxies with magnitudes fainter than -18 (van Zee et al., 2006). The dashed lines represent the mean value and standard deviation $(8.098 \pm 0.27)$ for the PNe of all the dwarf galaxies in the plot. For $12+\log (\mathrm{O} / \mathrm{H})$ : IC 10, Magrini \& Gonçalves (2009); SMC, Shaw et al. (2010); LMC, Leisy \& Dennefeld (2006); Sextans A and Sextans B, Magrini et al. (2005); Leo A, van Zee et al. (2006); NGC 3109, Peña et al. (2007); NGC 6822, Hernández-Martinez et al. (2009); M32, RM08; NGC 205, Richer \& McCall (2008) plus Gonçalves \& Magrini (2014); NGC 185, Gonçalves et al. (2012); Sagittarius, Zijlstra et al. (2006) and Otsuka et al. (2011); Fornax, Kniazev et al. (2007); and NGC 147, Gonçalves et al. (2007); M31, Jacoby \& Ciardullo (1999), Kwitter et al. (2012), Balick et al. (2013); Milky Way, Perinotto et al. (2004); M33, Magrini et al. (2009). Dwarf galaxies luminosities $\left(\mathrm{M}_{B}\right)$ are from Mateo (1998), those for the LMC and SMC are from Lee et al. (2003). $\mathrm{M}_{B}$ of the Galaxy, M31 and M33 are from van den Bergh (2007). Right: The PN MZR of the LG dwarf galaxies, showing $12+\log (\mathrm{O} / \mathrm{H})$ vs. stellar mass. Symbols and references are as in the left panel. Data for stellar masses were taken from the compilation by McConnachie (2012). The dashed line represents the mass-stellar metallicity relation of Kirby et al. (2013) to which we added a constant to match the oxygen metallicity, while the continuous line is the mean least square fit to our data. The direct method mass-metallicity relation for $M_{\star}$ stacks in Sloan SDSS Data Release 7 (DR7; Abazajian et al. 2009) (empty circles) from Andrews \& Martini (2013). The red solid curve shows the asymptotic logarithmic fit to the direct method measurements. The dashed red curve is an extrapolation of the fit.

smaller stellar mass range $\left(10^{7} \mathrm{M}_{\odot}\right.$ to $\left.10^{9} \mathrm{M}_{\odot}\right)$ in which we find that the MZR has a slope similar to that of the relation built with stellar metallicities. The MZR with $\mathrm{O} / \mathrm{H}$ seems to deviate only when reaching the stellar mass of the dIrr Leo A with $\mathrm{M}_{\star}<10^{7} \mathrm{M}_{\odot}$.

Fig. 3 also includes the comparison with the MZR for $M_{\star}$ stacks, obtained with Sloan SDSS Data (Andrews \& Martini 2013). Oxygen abundances are obtained adopting the direct method, since the [O III], [O II], [N II], and [S II] electron temperatures are measured from the stacked spectra - of $\sim 200,000$ star-forming galaxies in bins of 0.1 dex in stellar mass. Thus, Andrews \& Martini (2013)'s MZR is easily comparable with our results.

The above results point out a possible universality of the MZR for both dIrr and dSph galaxies. At variance with the previous results (see Richer \& McCall 1995; Gonçalves et al. 2007), which indicated a clearly different behaviour of dIrrs and dSphs, now these two classes of galaxies are compatible with a unique mass-metallicity relation. It is interesting to notice that both dIrrs and dSphs are consistent with global MZR of star forming galaxies, likely because chemical evolution is a function of stellar mass and its correlation with the total mass (baryonic and non-baryonic) of the galaxy. 


\section{References}

Amari, S., Hoppe, P., Zinner, E., \& Lewis R. S. 1995, Meteoritics, 30, 490

Abazajian, K. N., Adelman-McCarthy, J. K., Agüeros, M. A., Allam, S. S., \& Allende-Prieto, C., et al. 2009, ApJS, 182, 543

Andrews, B. H. \& Martini, P. 2013, ApJ, 765, 140

Balick, B., Kwitter, K. B., Corradi, R. L. M., \& Henry, R. B. C. 2013, ApJ, 774, 3

Gonçalves, D. R., Magrini, L., Leisy, P., \& Corradi, R. L. M. 2007, MNRAS, 375, 715

Gonçalves, D. R., Magrini, L., Martins, L. P., Teodorescu, A. M., \& Quireza, C. 2012, MNRAS, 419,854

Gonçalves, Denise R., Magrini, Laura, Teodorescu, Ana M., \& Carneiro, Carolina M. 2014, MNRAS, 444, 1705

Grebel, E. K., Gallagher, J. S., III, \& Harbeck, D. 2003 AJ, 125, 1926

Hernández-Martínez, L., Peña, M., Carigi, L., \& García-Rojas, J. 2009, A\&̊A, 505, 1027

Jacoby, G. H. \& Ciardullo, R. 1999, ApJ, 515, 169

Kingsburgh, R. L. \& Barlow, M. J. 1994, MNRAS, 271, 257

Kirby, E. N., Cohen, J. G., Guhathakurta, P., Cheng, L., Bullock, J. S., \& Gallazzi, A. 2013, ApJ, 779, 102

Kniazev, A. Y., Grebel, E. K., Pustilnik, S. A., Pramskij, A. G., \& Zucker, D. B. 2005, AJ, 130, 1558

Kniazev, A. Y., Grebel, E. K., Pustilnik, S. A., \& Pramskij, A. G. 2007, A\&A, 468, 121

Kormendy, J. \& Djorgovski, S. 1989, ARA\&A, 27, 235

Kormendy, J. \& Bender, R. 2012, ApJS, 198,2

Kwitter, K. B., Lehman, E. M. M., Balick, B., \& Henry, R. B. C. 2012, ApJ, 753, 12

Lee, H., McCall, M. L., Kingsburgh, R. L., Ross, R., \& Stevenson, C. C. 2003, AJ, 125, 146

Leisy, P., Dennefeld, M. 2006, A\& $A, 456,451$

McConnachie, A. W., 2012 AJ, 144, 4

Magrini, L., Leisy, P., Corradi, R. L. M., Perinotto, M., Mampaso, A., \& Vilchez, J. M. 2005, $A \& A, 443,115$

Magrini, L., Leisy, P., Corradi, R. L. M., Perinotto, M., Mampaso, A., \& Vilchez, J. M. 2005, $A \mathscr{E} A, 443,115$

Magrini, L. \& Gonçalves, D. R. 2009, MNRAS, 398, 280

Magrini, L., Stanghellini, L., \& Villaver, E. 2009, ApJ, 696, 729

Mateo, M. L. 1998, ARAEAA, 36, 435

Otsuka, M., Meixner, M., Riebel, D., Hyung, S., Tajitsu, A., \& Izumiura, H. 2011, ApJ, 729, 39

Peña, M., Stasińska, G., \& Richer, M. G. 2007, A\&A, 476, 745

Perinotto, M., Morbidelli, L., \& Scatarzi, A. 2004, MNRAS, 349, 793

Péquignot, D., Walsh, J. R., Zijlstra, A. A., \& Dudziak, G. 2000, A\&A, 361, L1

Richer, M. G. \& McCall, M. L. 1995, ApJ, 445, 642

Richer, M. G. \& McCall, M. L. 2007, ApJ, 658,328

Richer, M. G. \& McCall, M. L. 2008, ApJ, 684, 119

Salaris, M. \& Girardi, L. 2005, MNRAS, 357, 669

Shaw, R. A., Lee, T.-H., Stanghellini, L., Davies, J. E., García-Hernández, D. A., et al. 2010, ApJ, 717, 562

Skillman, E. D., Kennicutt, R. C., \& Hodge, P. W. 1989, ApJ, 347, 875

Tremonti, C. A., Heckman, T. M., Kauffmann, G., Brinchmann, J., Charlot, S., et al. 2004, ApJ, 613, 898

Weisz, Daniel R., Dolphin, Andrew E., Skillman, Evan D., Holtzman, Jon, Gilbert, Karoline M., Dalcanton, Julianne J., \& Williams, Benjamin F. 2014, ApJ, 789, 147

van den Bergh, S., 2007, in The Galaxies of the Local Group, Cambridge: Cambridge University Press 2007

van Zee, L., Skillman, E. D., \& Haynes, M. P. 2006, ApJ, 637, 269

Zijlstra, A. A., Gesicki, K., Walsh, J. R., Péquignot, D., van Hoof, P. A. M., \& Minniti, D. 2006, MNRAS, 369, 875 